\title{
RELATION OF GUTZWILLER VARIATIONAL STATE WITH MATRIX MODEL
}

\author{
A. KOPER AND M. MUCha \\ Institute of Physics, A. Mickiewicz University \\ Matejki 48/49, 60-769 Poznań, Poland
}

\begin{abstract}
It is shown that mean values of electron operators in variational Gutzwiller state are equal to mean values of corresponding classical quantities calculated by means of a hermitian matrix model. In cases with small number of electrons in the system this property enables exact calculation of the mean values. In case of large number of electrons a simple and effective Monte Carlo method is formulated (within matrix model).
\end{abstract}

PACS numbers: 71.10.-w, 71.27.+a, 11.10.-z, 02.50.Ng, 02.70.Lq

\section{Introduction}

The Gutzwiller variational ground state $\left|\Phi_{\mathrm{G}}\right\rangle=\prod_{i}\left[1-\left(1-g_{i}\right) n_{i \uparrow} n_{i \downarrow}\right]|\Phi\rangle$ is commonly known to enable more precise calculation of electron ground state energy than that of product state $|\Phi\rangle$ (for example Hartree-Fock state) [1]. The quantities $g_{i} \in[0,1]$ are the variational parameters. The operators $n_{i \sigma}$ can be expressed by fermion creation and annihilation operators $n_{i \sigma}=a_{i \sigma}^{\dagger} a_{i \sigma}, i=1, \ldots, N, N-$ number of sites in the system. The calculation of quantum operators mean values $\langle\widehat{T}\rangle_{\mathrm{G}}=\left\langle\Phi_{\mathrm{G}}|\widehat{T}| \Phi_{\mathrm{G}}\right\rangle /\left\|\Phi_{\mathrm{G}}\right\|^{2}$ is troublesome and that is why alternative methods are being searched for. We show that the mean values $\langle\widehat{T}\rangle_{G}$ can be calculated relatively easy using a version of the so-called hermitian matrix model (HMM) which is a classical model. This model is connected with the theory of disordered systems (cf. [2]). We confine ourselves to the single particle operators $\hat{T}$ since in the context of our calculations e.g. in Hubbard model it suffices (operators $n_{i \uparrow}$ and $n_{i \downarrow}$ commute).

In order to simplify notation we assume that the creation (annihilation) operators for spins $\sigma=\uparrow$ are labelled with numbers from 1 to $N$, and for spins $\sigma=\downarrow$ with numbers $N+1$ to $2 N$. In this notation we have $\left|\Phi_{\mathrm{G}}\right\rangle=\prod_{i}[1-$ $\left.\left(1-g_{i}\right) n_{i} n_{N+i}\right]|\Phi\rangle$, where $m$-particle product state $|\Phi\rangle=\prod_{\alpha \in A} c_{\alpha}^{\dagger}|0\rangle$, the creation operators $c_{\alpha}^{\dagger}$ are expressed by $c_{\alpha}^{\dagger}=\sum_{i=1}^{2 N} \Phi_{\alpha, i} a_{i}^{\dagger}, A$ is a set of $m$ different quantum numbers (e.g. set of vector pairs quasi-momentum and spin $(k, \sigma))$. The trial state $|\Phi\rangle$ is constructed of the single particle state vectors $\left(\Phi_{\alpha, 1}, \ldots, \Phi_{\alpha, 2 N}\right)$. We shall identify this state with matrix $\Phi^{\prime}$ transposed to $\Phi=\left(\Phi_{\alpha, i}\right)_{A \times 2 N}$. If $|\Psi\rangle$ is also a $m$-particle product state the commonly known Slater formula $\langle\Psi \mid \Phi\rangle=\operatorname{det}\left(\bar{\Psi} \Phi^{\prime}\right)$ holds (bar denotes complex conjugate). This is an important formula since it says 
that the quantity $\langle\Psi|\widehat{T}| \Phi\rangle$ can be easily calculated when and only when the operator $\widehat{T}$ is a single-particle one: $\widehat{T}=\sum_{i, j=1}^{2 N} T_{i j} a_{i}^{\dagger} a_{j}$. It can be shown that the following formula holds for the so-called $\tau$-functions: $\tau(\beta) \stackrel{\mathrm{df}}{=}\left\langle\Psi\left|\mathrm{e}^{\beta \widehat{T}}\right| \Phi\right\rangle=\operatorname{det}\left(\bar{\Psi} \mathrm{e}^{\beta T} \Phi^{\prime}\right)$, where $\mathrm{e}^{\beta T}$ on the right hand side denotes exponent of the matrix $T=\left(T_{i j}\right)$. The parameter $\beta$ (Schwinger source) can be used for differentiation. Therefore, we can write $\langle\Psi|\widehat{T}| \Phi\rangle=\left.\frac{\partial \tau(\beta)}{\partial \beta}\right|_{\beta=0}=\operatorname{tr}\left(\bar{\Psi} T \Phi^{\prime} M^{-1}\right) \operatorname{det}(M)$, where $M=\bar{\Psi} \Phi$. Proof of the formula for $\tau$-functions (cf. [3]) is based on noticing the fact that $\mathrm{e}^{\beta \widehat{T}}$ is in principle a rotation operator whose action can be easily calculated since $\mathrm{e}^{\beta \widehat{T}}|\Phi\rangle=\prod_{\alpha \in A}\left(\mathrm{e}^{\beta \widehat{T}} c_{\alpha}^{\dagger} \mathrm{e}^{-\beta \widehat{T}}\right)|0\rangle$.

Difficulty of calculation of analogous scalar products in the case of Gutzwiller states consists in the fact that they are not product states because $\left|\Phi_{\mathrm{G}}\right\rangle=\mathrm{e}^{\widetilde{\Gamma}}|\Phi\rangle$, $\widetilde{\Gamma}=\sum_{\mathrm{i}=1}^{\mathrm{N}} \eta_{\mathrm{i}} \mathrm{n}_{\mathrm{i}} \mathrm{n}_{\mathrm{N}+\mathrm{i}}$, where $\eta_{i}=\log \left(g_{i}\right)$. The remedy is Hubbard transformation applied to the $\mathrm{e}^{\widetilde{\Gamma}}$ operator.

\section{From Hubbard transformation to matrix model}

We shall apply the so-called discrete version of Hubbard transformation: $\mathrm{e}^{-\eta_{i} n_{i} n_{N+i}}=\frac{1}{2} \sum_{x_{i} \in\{ \pm 1\}} \mathrm{e}^{\gamma_{i} n_{i}+\gamma_{N+i} n_{N+i}}$, where $\gamma_{i}=-J_{i} x_{i}-\eta_{i} / 2, \gamma_{N+i}=J_{i} x_{i}-$ $\eta_{i} / 2$ and $J_{i}$ can be found from $\cosh \left(J_{i}\right)=\mathrm{e}^{-\eta_{i} / 2}$. In consequence $\left|\Phi_{\mathrm{G}}\right\rangle=$ $\frac{1}{2^{N}} \sum_{x_{1}, \ldots, x_{N}} \mathrm{e}^{\widehat{\Gamma}(x)}|\Phi\rangle, \widehat{\Gamma}(x)=\sum_{i=1}^{2 N} \gamma_{i} n_{i}$. It can be shown, exactly in the same manner as for the $\tau$-functions, that the state $\mathrm{e}^{\widehat{\Gamma}(x)}|\Phi\rangle$ is a product state and can be expressed by formula $\mathrm{e}^{\widehat{\Gamma}(x)}|\Phi\rangle=|\Phi \Gamma(x)\rangle$, where on the right hand side we have product of two matrices $\Phi \Gamma(x)$, the second matrix is diagonal $\Gamma(x)=$ $\operatorname{diag}\left(\mathrm{e}^{\gamma_{1}}, \ldots, \mathrm{e}^{\gamma_{2 N}}\right)$. As a result of our considerations we obtain

$$
\left|\Phi_{\mathrm{G}}\right\rangle=\frac{1}{2^{N}} \sum_{x_{1}, \ldots, x_{N}}|\Phi \Gamma(x)\rangle
$$

- Gutzwiller state is a mean value of random product states $|\Phi \Gamma(x)\rangle$. The random variable $x=\left(x_{1}, \ldots, x_{N}\right)$ takes $2^{N}$ different discrete values with the same probability $p=1 / 2^{N}$. Therefore, we can easily show that

$$
\left\langle\Phi_{\mathrm{G}}|T| \Phi_{\mathrm{G}}\right\rangle=\frac{1}{2^{2 N}} \sum_{x, y} \operatorname{tr}\left[T(M) M^{-1}(x, y)\right] \operatorname{det}[M(x, y)]
$$

holds, where $M(x, y)=\bar{\Phi} \Gamma(x) \Gamma(y) \Phi^{\prime}, T(M)=\bar{\Phi} \Gamma(x) T \Gamma(y) \Phi^{\prime}$. The matrix $M(x, y)$ is hermitian and has dimension of $m \times m$ ( $m$ - number of electrons), it can be also shown that all its eigenvalues (for parameters $g_{i} \in[0,1]$ ) are greater than zero, so the formula (2) has the following probabilistic interpretation. Let $\Omega(g)$ be a set of all hermitian matrices $M(x, y)$ (the matrices depend on Gutzwiller parameters $g$ ). Mean value of arbitrary single particle operator $\widehat{T}$ taken over variational Gutzwiller state $\left|\Phi_{G}\right\rangle$ is equal to mean in the matrix model taken over hermitian matrices $\Omega(g)$

$$
\langle\widehat{T}\rangle_{\mathrm{G}}=\frac{\sum_{M \in \Omega} \operatorname{tr}\left[T(M) M^{-1}\right] \mathrm{e}^{-\operatorname{tr} V(M)}}{\sum_{M \in \Omega} \mathrm{e}^{-\operatorname{tr} V(M)}}
$$


where the potential $V$ in the matrix model equals $V(M)=-\log [\operatorname{det}(M)]=$ $\sum_{n \geq 1}(-1)^{n}(M-1)^{n} / n$. It would be sensible to call that model the Gutzwiller matrix model (GMM). Probabilistic correlations in GMM cover far distant matrix elements $M_{\mu v}$ (all electrons in Gutzwiller state are strongly correlated with each other) in contrast to HMM (cf. [2]). Because of the fact when the number of electrons $m$ is large it would be unrealistic to expect exact results for mean values $\langle\widehat{T}\rangle_{\mathrm{G}}$ in GMM. In such case the calculations can be carried out by means of Monte Carlo method (particularly suitable for that purpose is the formula (2), which reminds thermodynamical mean in generalized Ising model with Hamiltonian $H(x, y)=-\log \operatorname{det}[M(x, y)])$. If the number of electrons is not large $(m \leq 12)$ then the exact calculations in GMM are possible thanks to $\tau$-functions with the use of computer software packages for analytical transformations [4]. Simple applications of the described formalism are shown in Figs. 1,2 and more complicated ones in [5]. Analogous calculations can be carried out within other quantum models e.g. extended Hubbard model, Heisenberg model and $t-J$ model (there will be bigger and more complicated matrices $\Phi, T, \Gamma$ and $M$ ) [4].

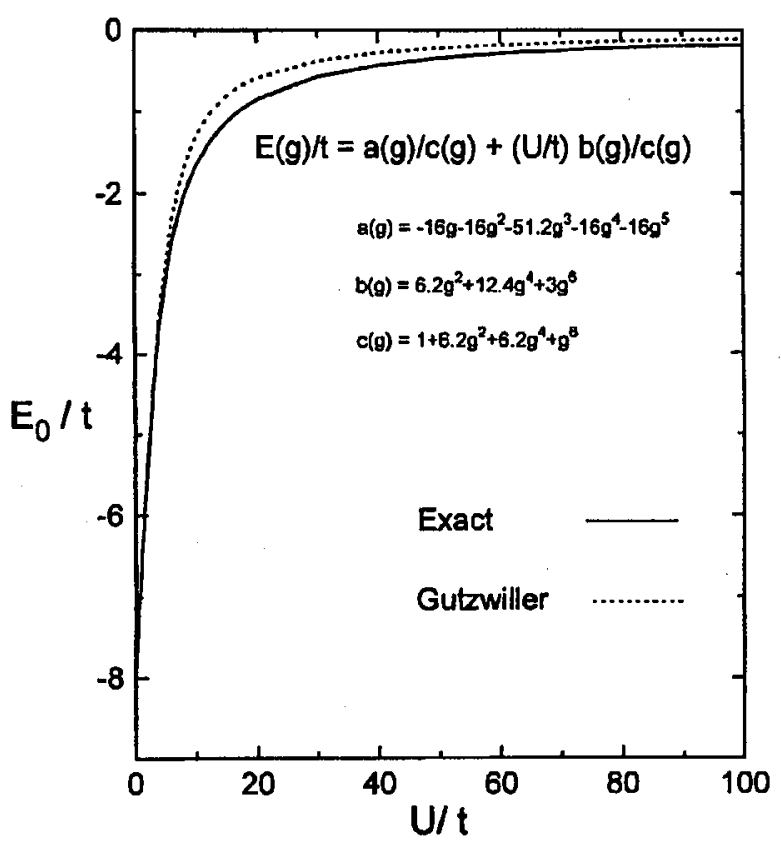

Fig. 1. Comparison of exact energy value of $\pi$-electrons ground state Hubbard model in benzene ring $(N=m=6)$ and energy calculated for the same system by means of the Gutzwiller variational state $\left|\Phi_{G}\right\rangle$, when the trial state is a tight binding state. The mean values in the Gutzwiller state were calculated by means of $\tau$-functions using computer analytic transformations software. The optimal energy in the Gutzwiller state was found by minimizing the function $E(g) / t$. 


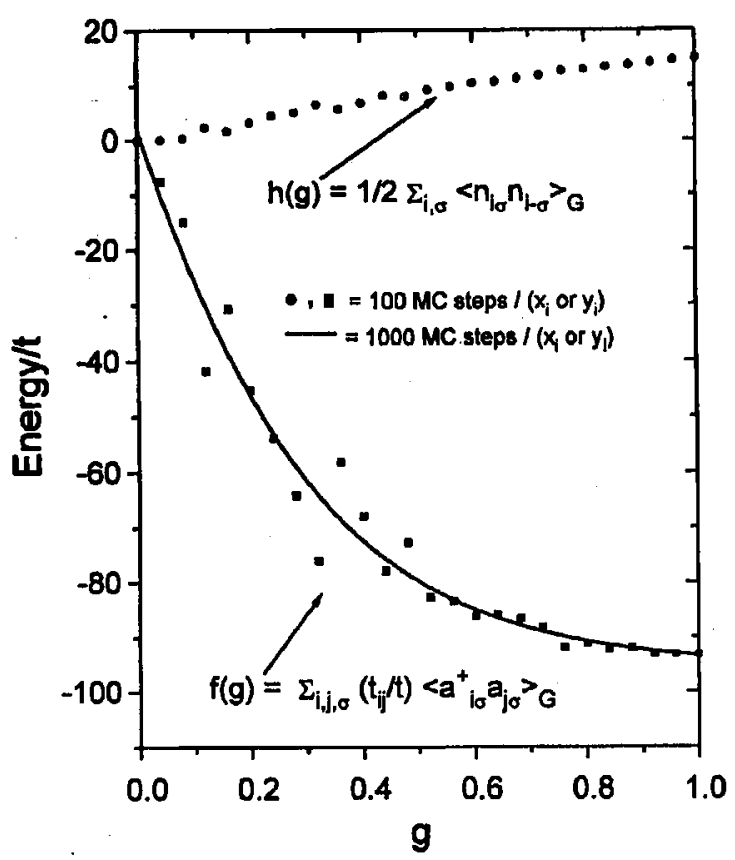

Fig. 2. An example of application of Metropolis et al. algorithm for Monte Carlo calculation of mean values in variational Gutzwiller ground state $\left|\Phi_{G}\right\rangle$ describing $\pi$-electrons in $\mathrm{C}_{60}$ molecule $(N=m=60)$, when the trial state $|\Phi\rangle$ is tight binding state. The optimal energy in the Gutzwiller state was found by minimizing the function $E(g) / t=f(g)+(U / t) h(g)$.

This work is supported by the Committee for Scientific Research under grant No. 3 P408 00104.

\section{References}

[1] M.C. Gutzwiller, Phys. Rev. Lett. 10, 159 (1963); Phys. Rev. 134, A923 (1964); Phys. Rev. 137, A1726 (1965).

[2] M.L. Mehta, Random Matrices, Academic, New York 1991.

[3] S. Kharchev, A. Marshakov, A. Mironov, A. Zabrodin, Nucl. Phys. B 366, 569 (1991).

[4] A. Koper, M. Mucha, to be published.

[5] A. Koper, M. Mucha, Acta Phys. Pol. A 92, (1997), Proc. of this Conf. (Part III). 